\title{
Response-key input via the IBM PC/XT/AT's parallel printer port
}

\author{
E. C. DALRYMPLE-ALFORD \\ University of Guelph, Guelph, Ontario, Canada
}

A simple and inexpensive way to connect response keys to the IBM PC/XT/AT's parallel printer port is presented.

Recent writers in this journal (e.g., Crosbie, 1990; Segalowitz \& Graves, 1990) have examined the keyboard, mouse, and game port as response devices for the IBM PC. From the information given in Eckel (1987), Hogan (1988), and Sargent and Shoemaker (1984), one can arrive at another convenient and inexpensive source of input to the computer, namely via the parallel printer port, which permits connecting up to 15 keys (including relay contacts), without the need for an external power supply.

The simplest use of the parallel port occurs when up to four keys are connected. All that this requires is the connection of switches between any of Pins 1, 14, 16, and 17, and ground Pins 18-25 of the DB-25 male connector for that port (see Figure 1; pin numbers will be found on the DB- 25 connector). There is no possibility of damage, for no external power supply is involved. Pins $1,14,16$, and 17 are the only ones that can receive input in this simple way. There is the added convenience of their affecting the lower four bits of the same I/O address, which is 037AH if input is to printer port LPT1 and 027AH if input is to printer port LPT2. Examples of the appropriate commands given below assume that LPT1 (i.e., Port 037AH) is being used.

Before the status of the switches can be determined by reading Port $037 \mathrm{AH}$, the port should be initialized by sending the value $\mathrm{F} 4 \mathrm{H}$ to it. This sets the $\mathrm{I} / \mathrm{O}$ port to respond to input, and it only needs to be done once. The Turbo Pascal command for this is

$$
\text { Port }[\$ 037 \mathrm{~A}]:=\$ F 4
$$

Pins 1, 14, 16, and 17 connect to Bits $0,1,2$, and 3, respectively, of the $\mathrm{I} / \mathrm{O}$ port. Closing any of the switches SW1, SW2, or SW4 will ground the corresponding pin and set its bit to 1; open switches will set the bit to 0 . Input to Pin 16 is inverted, so that closing SW3 (i.e., grounding that pin) will set Bit 3 to 0 ; opening SW3 will set it to 1 . There is no internal latching of input, so the values of the bits change as switches are open and closed.

This paper benefited from the helpful comments of the journal's reviewers. Correspondence should be addressed to E. C. DalrympleAlford, Department of Psychology, University of Guelph, Guelph, Ontario N1G 2W1, Canada.

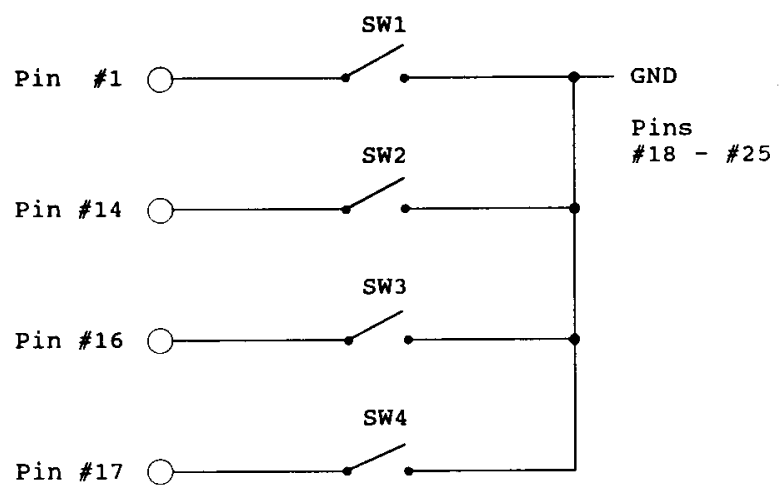

Figure 1. Connecting four input switches to the parallel port DB25 connector.

This means that normally closed as well as normally open switches may be used.

The Turbo Pascal instructions for reading the port for the status of the switches are given below, incorporated in a loop that waits for a switch closure:

$$
\begin{aligned}
& \text { REPEAT } \\
& \text { Response := Port[\$037A] } \\
& \text { UNTIL (Response }<>\text { \$F4); }
\end{aligned}
$$

(\$F4 is the reading while all switches are open). Response may be tested subsequently to determine which switch was closed. Such loops work very well when the switch closures are generated manually, for the duration of the response is substantially greater than the time to execute a loop; there is no need to introduce latching of the input. It may be necessary in some situations to deal with the problem of switch-contact bounce (see Genovese, 1988).

The following Turbo Pascal code gives an example of how the status of Switch SW1 (Bit 1) may be checked by testing the value read into Response:

$$
\begin{aligned}
& \text { IF (Response AND } \$ 01)=\$ 01 \text { THEN } \\
& \text { SW1closed := TRUE } \\
& \text { ELSE } \\
& \quad \text { SW1closed := FALSE; }
\end{aligned}
$$

For the other switches, the test would be 
for SW2 (Bit 1, Pin 14): (Response AND \$02) $=\$ 02$ for SW3 (Bit 2, Pin 16): (Response AND \$04) $=\$ 00$ for SW4 (Bit 3, Pin 17): (Response AND \$08) $=\$ 08$

(In the case of SW3, recall that grounding Pin 16 changes Bit 3 from 1 to 0 .)

By using switches that simultaneously ground more than one of the Pins $1,14,16$, and 17, we can increase the number of response switches to 15 (i.e., $4^{2}-1$ ). Beyond

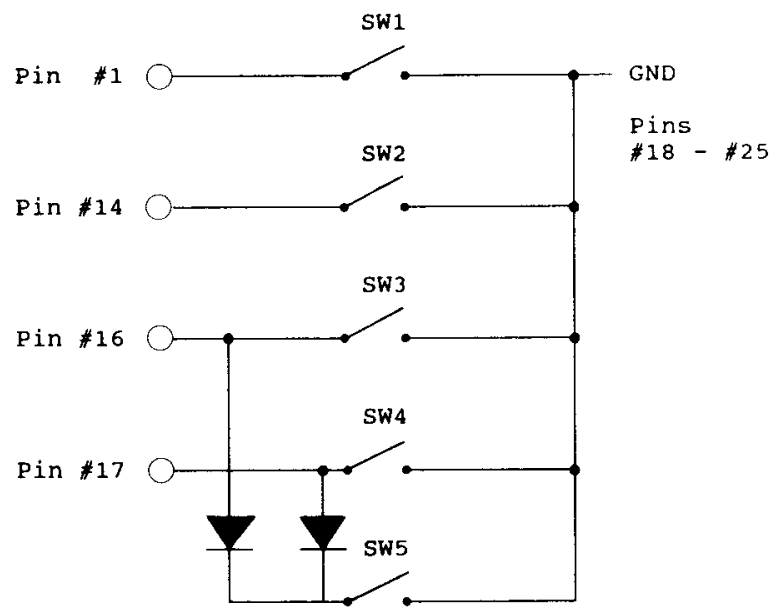

Figure 2. Illustration of the use of diodes when more than four switches are connected to the parallel port. (Switch SW5 grounds Pins 16 and 17.) the four switches connected as shown in Figure 1, diodes (e.g., 1N4148) are required to isolate the operations of switches connected to the same input pins. This is illustrated in Figure 2. When SW5 is closed, it grounds Pins 16 and 17, causing Bit 2 to be cleared and Bit 3 to be set. The status of switches connected in this way may thus be determined by checking Bits 1-4 as described above.

In summary, to connect response keys to the parallel printer port is a very simple matter in terms of both hardware and software, and it provides a quick and inexpensive way to obtain experimental input.

\section{REFERENCES}

Crosbie, J. (1990). The Microsoft mouse as a multipurpose response device for the IBM PC/XT/AT. Behavior Research Methods, Instruments, \& Computers, 22, 305-316.

ECKEL, B. (1987). A programmer's guide to the parallel port. Turbo Technix, 1, 74-79.

Genovese, R. F. (1988). A strategy for microcomputer-controlled measurement of responses in the behavioral laboratory. Behavior Research Methods, Instruments, \& Computers, 20, 1-5.

HogAN, T. (1988). The programmer's PC sourcebook. Redmond, WA: Microsoft Press.

SARgent, M., Shoemaker, R. L. (1984). The IBM Personal Computer from the inside out. Reading, MA: Addison-Wesley.

Segalowitz, S. J., \& Graves, R. E. (1990). Suitability of the IBM $\mathrm{XT}, \mathrm{AT}$, and PS/2 keyboard, mouse, and game port as response devices in reaction time paradigms. Behavior Research Methods, Instruments, \& Computers, 22, 283-289.

(Manuscript received September 4, 1990; revision accepted for publication June 7,1991 .) 